\title{
Segmentation automatique des images pour la planification dosimétrique en radiothérapie.
}

\author{
Automatic image segmentation for treatment planning in radiotherapy

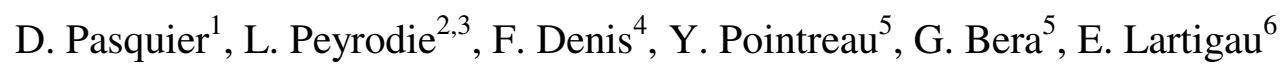

1) Centre Galilée, Polyclinique de la Louvière, 69 rue de la Louvière, 59000 Lille, France

2) Ecole des Hautes Etudes d'Ingénieur, 13 rue de Toul, 59046 Lille

3) Laboratoire d'Automatique, Génie Informatique et Signal (LAGIS), Cité Scientifique, 59651 Villeneuve d'Ascq

4) Centre Jean Bernard, 9 rue Beauverger, 72000 Le Mans

5) Clinique d'Oncologie Radiothérapie, Centre Henry-S-Kaplan, CHU Bretonneau, 37044 Tours cedex 9, France

6) Département universitaire de radiothérapie, Centre O. Lambret, Université Lille II, 3 rue F. Combemale 59000 Lille

Segmentation automatique des images en radiothérapie

Auteur correspondant : D. Pasquier, Centre Galilée, Polyclinique de la Louvière, 69 rue de la Louvière, Lille. E-mail: davidpasquier@free.fr 
L'essor de la radiothérapie conformationnelle et de la radiothérapie guidée par l'image a pour corollaire l'accroissement du temps consacré à la délinéation des volumes d'intérêt, qui est également le siège d'une variabilité inter et intraobservateur. Les développements informatiques et du traitement d'images ont permis des progrès dans l'automatisation partielle ou totale de ces tâches. Cet article détaille les grands principes de la segmentation d'images appliquée à la radiothérapie, ses applications ainsi que ses résultats les plus récents dans un contexte clinique.

Mots clefs: Traitement d'images, Segmentation automatique, Radiothérapie conformationnelle, Radiothérapie guidée par l'image 
One drawback of the growth in conformal radiotherapy and image-guided radiotherapy is the increased time needed to define the volumes of interest. This also results in inter- and intraobserver variability. However, developments in computing and image processing have enabled these tasks to be partially or totally automated. This article will provide a detailed description of the main principles of image segmentation in radiotherapy, its applications and the most recent results in a clinical context.

Key words: Computer Assisted Image Processing, Automatic segmentation, Conformal radiotherapy, Image-guided radiotherapy 


\section{$\underline{\text { Introduction }}$}

La radiothérapie conformationnelle et la radiothérapie guidée par l'image impliquent un accroissement considérable du temps nécessaire à la délinéation des volumes d'intérêt, ce qui rend souhaitable une automatisation au moins partielle de ces tâches. Cette délinéation est également le siège d'une variabilité inter et intra-observateur, qui tend à être diminuée par les recommandations internationales et les atlas de radioanatomie comme SIRIADE (site de radioanatomie et d'aide à la délinéation) [55]. Dans de nombreux domaines médicaux une automatisation du traitement des données a été développée, comme l'analyse des signaux électrocardiographiques, électroencéphalographiques, ou encore l'imagerie cardiaque et des pathologies inflammatoires neurologiques. Les développements informatiques et du traitement d'images ont permis plus récemment l'extension de ces techniques d'automatisation à la radiothérapie. Cet article traite des méthodes et résultats de la segmentation d'images morphologiques destinées à la planification dosimétrique (tomodensitométrie et remnographie). Il détaille les grands principes de la segmentation d'images appliquée à la radiothérapie, ses méthodes d'évaluation, ses applications, et ses résultats les plus récents dans un contexte clinique.

\section{1) Méthodes de segmentation automatique}

La segmentation d'image est une opération de traitement d'images dont la finalité est de rassembler des pixels entre eux suivant des critères prédéfinis, le plus souvent les niveaux de gris ou la texture. Les pixels sont ainsi regroupés en régions, qui constituent une partition de l'image. Il existe de nombreuses méthodes de segmentation, que l'on peut schématiquement regrouper en deux principales catégories: segmentation par régions et segmentation par contours.

\section{1-1) Segmentation par régions}

La méthode la plus basique pour définir une région d'intensité uniforme est le seuillage des niveaux de gris. Une autre méthode consiste à diviser successivement l'image jusqu'à ce que les voxels des sous-régions satisfassent à des propriétés de similitude. Les sous-régions 
voisines sont ensuite examinées afin d'effectuer d'éventuelles fusions (méthode de divisionfusion ou «split and merge »). Cet algorithme a pour inconvénient d'intervenir à un niveau moindre de détails que d'autres algorithmes, comme par exemple l'agglomération de voxels («region growing»). Cette méthode consiste à rattacher à un voxel de départ les voxels voisins respectant des fonctions identiques comme une faible variation d'intensité.

\section{1-2) Segmentation par contours}

La détection de contours consiste à rechercher les primitives au sein de l'image. Elles sont de plusieurs types, et peuvent consister en des lignes, des courbes ou des contours (détection des gradients d'intensité). Des filtres permettent de mesurer des sauts d'intensité dans l'image. Un seuillage permet d'éliminer des voxels au sein desquels la variation d'intensité est trop faible pour qu'ils puissent appartenir à un contour. Poussé au maximum, un seuillage peut donner une image binaire au sein de laquelle tout voxel de l'image est un point de contour. Des réglages intermédiaires permettent de conserver certaines valeurs d'intensité. Un autre type de seuillage, par hystérésis, fait intervenir deux seuils : un seuil bas et un seuil haut. Tout voxel dont le module de contour est supérieur au seuil haut est considéré comme appartenant à un contour; les voxels voisins sont examinés dans la direction du contour et ajoutés au contour si leur valeur est supérieure au seuil bas.

Les caractéristiques d'un filtre détecteur de contour doivent permettre une détection et une localisation correctes, et une réponse unique par contour. Parmi les plus utilisés, citons les filtres de Canny [7] et de Deriche [17]. Des opérations morpho-mathématiques peuvent être appliquées afin d'améliorer les contours, comme une érosion ou une dilatation. Une ouverture consiste à appliquer une érosion suivie d'une dilatation: les petites structures et détails disparaissent lors de l'érosion et ne sont pas recréés lors de la dilatation. Une ouverture permet ainsi d'éliminer des segments courts et bruités. Une fermeture consiste en une succession d'une dilatation et d'une érosion. Les structures proches fusionnent avec le contour lors de la dilatation et seules les fusions ponctuelles disparaissent lors de l'érosion, permettant d'obturer ainsi les courts défects des contours. 


\section{1-3) Recalage élastique avec atlas, modèles déformables}

D'autres méthodes ont été plus récemment développées et appliquées à la segmentation d'images en radiothérapie, avec des résultats prometteurs, comme le recalage élastique avec un atlas et l'application de modèles déformables.

La première méthode consiste à construire un atlas qui va comporter les différentes structures à délinéer; cet atlas peut être créé à partir d'images de patients ou d'images synthétiques. Un recalage élastique, avec déformation de l'atlas, est ensuite appliqué aux images à segmenter. Le recalage élastique est souvent précédé d'un recalage rigide. Il existe différents algorithmes de recalage élastique, qui vont déformer les structures de l'atlas, selon un mode non linéaire, de façon globale ou locale. Ces algorithmes ne seront pas détaillés ici, mais nous pouvons classer les méthodes de recalage en deux grandes catégories [36]:

- L'approche géométrique, qui repose sur la recherche de primitives caractéristiques au sein des images puis sur l'appariement de ces caractéristiques. Les primitives peuvent être des points, des lignes ou des surfaces, et peuvent être sélectionnées manuellement ou automatiquement. En imagerie médicale et notamment cérébrale les primitives les plus souvent employées sont les modèles surfaciques. Les méthodes d'appariement les plus souvent utilisées sont les splines de plaque mince et la méthode des plus proches voisins itératifs («Iterative Closest Point ») [1]

- L'approche iconique est plus générale que l'approche géométrique, en étant basée sur l'ensemble des informations des images recalées, et ne nécessite pas de prétraitement des images. La mesure de similarité entre les deux séries d'images peut faire intervenir notamment les critères d'erreur quadratique, le coefficient de corrélation, la variance d'intensité, l'entropie (information mutuelle).

La technique des atlas se heurte à la grande variabilité de la forme et du volume des structures à délinéer, notamment pour les localisations pelviennes. Cette technique est utilisée pour la segmentation des structures cérébrales, pelviennes ou de la tête et du cou $[5,15,16,25,47]$. Pour certaines localisations comme la tête et le cou, de multiples atlas ont été créés, afin que l'utilisateur puisse choisir celui qui se rapproche le plus du patient dont les images doivent être segmentées [11]. 
La méthode des modèles déformables est basée sur l'évolution d'un contour fermé et permet de s'affranchir partiellement des problèmes de bruits parasites et de chaînage. Cette technique, dont le principe est de faire évoluer un contour initial vers une position d'équilibre, peut être assimilée à une segmentation par contours. Le modèle peut être élaboré à partir d'une forme mathématique (sphère, ellipse) ou intégrer des connaissances relatives au volume d'intérêt à segmenter, comme la forme ou la texture. La première étape consiste à placer le modèle au sein de l'image à segmenter. Le processus de déformation, souvent selon la normale, est lié à la minimisation d'une fonctionnelle d'énergie. Les forces dépendent des informations présentes dans l'image (gradient, intensité...) et des propriétés de régularité des contours. La déformation va se faire sous l'action de plusieurs forces : une énergie propre ou interne, assimilable à une énergie mécanique de tension et contrôlant l'aspect du contour, et une énergie externe introduite pour répondre aux contraintes spécifiques du volume d'intérêt et attirant le contour vers l'objet dont on cherche à déterminer les frontières $[26,49]$. Le contour actif va se déformer pour rechercher la position d'énergie minimale en repectant au mieux les contraintes. Cette fonctionnelle d'énergie peut être représentée sous la forme de l'équation :

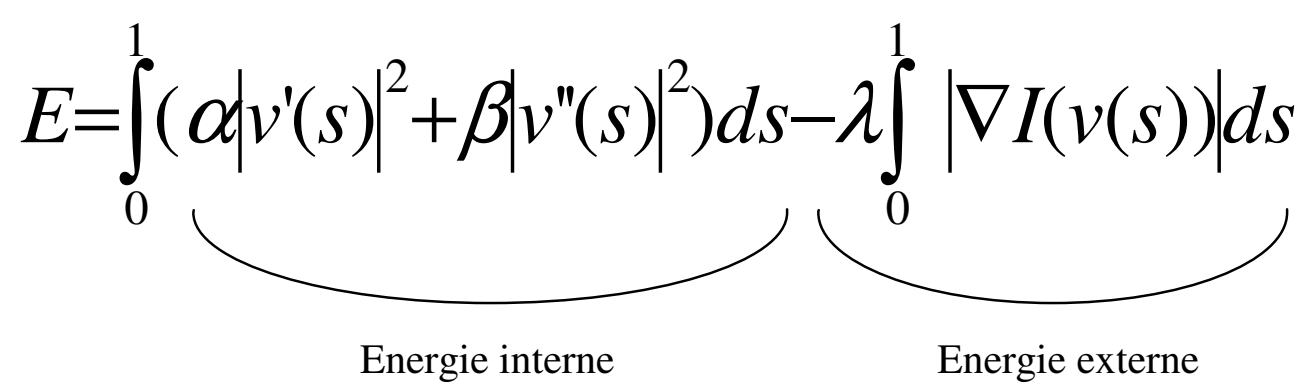

dans laquelle $\alpha$ et $\beta$ sont les coefficients d'élasticité et de rigidité de la courbe. Les contraintes liées à l'énergie interne des modèles peuvent limiter leur flexibilité géométrique et rendre plus difficile leur application à des volumes présentant d'importantes irrégularités [32]. Diverses méthodes ont été proposées pour améliorer et mieux automatiser le processus de segmentation du contour déformable. Cohen et al. [13] et Terzopoulos et al. [49] ont utilisé une force d' «inflation interne » rendant le modèle moins sensible aux conditions initiales de placement du modèle au sein de l'image. De nombreux auteurs ont intégré au modèle déformable des connaissances à priori, comme la taille, la forme, les variations de forme attendues, la localisation et l'orientation du volume d'intérêt, ou encore la texture [18]. La limite des modèles déformables est leur caractère surfacique et l'absence de modélisation de la partie centrale des objets, ce qui rend plus difficile leur représentation et l'établissement de relations 
inter-objets. Cette technique est employée pour la segmentation des volumes d'intérêt pelviens (prostate, vessie, têtes fémorales) et de la tête et du cou [2,3,21,28,37,39].

L'équipe de Pizer et Chaney a contribué au développement des modèles déformables et à leur utilisation en radiothérapie, en particulier en décrivant les «medial representations » ou m-reps $[33,40]$. Les $m$-reps sont des modélisations d'objets solides, à la fois de leur structure interne et de leur surface. La structure interne est modélisée par un échantillonnage du plan médian grâce à des atomes, reliés entre eux par des rayons. Chaque atome contient comme informations : sa position spatiale, la longueur des rayons qui le relient aux atomes voisins ou à la surface, l'orientation des rayons, et sa distance avec le bord pour les atomes situés près des arêtes. Les volumes d'intérêt cylindriques comme le rectum sont représentés par des $m$ reps en forme de tube comprenant une seule chaîne d'atomes comportant des rayons d'inégales longueurs afin de modéliser les sections non circulaires (Figure 1). L'architecture des m-reps avec leurs interactions étroites entre les atomes permettrait une meilleure représentation des propriétés géométriques des formes et de leur variation [11]. Les m-reps contiennent également des connaissances a priori sur l'apparence des objets, grâce à une analyse statistique des images à la fois à l'intérieur et à l'extérieur des modèles, ce qui permet à un objet d'appréhender son environnement et les structures voisines. Cette méthode a été appliquée à la segmentation des reins, de la prostate, de la vessie et du rectum [33,40,42]. Les résultats sont encourageants avec néanmoins un certain degré d'interaction, l'utilisateur devant par exemple déterminer manuellement les limites du volume d'intérêt à segmenter sur 3 coupes pour la prostate et le rectum.

\section{2) Evaluation des outils de segmentation automatique}

La littérature traitant du développement et de l'évaluation des algorithmes appliqués à des images tests à type de fantômes est très abondante et son étude dépasse le cadre de notre article, qui traite des résultats les plus récents dans le contexte d'une évaluation clinique ou «pré-clinique ». De nombreuses méthodes sont utilisables pour l'évaluation des résultats obtenus, et leur multiplicité dans la littérature rend difficile la comparaison des différents outils de segmentation automatique. La plupart des auteurs utilise les distances linéaires entre les contours définis manuellement et de façon automatique comme la distance radiale, la distance entre deux points les plus proches, la distance à partir de la normale de la surface et la distance de Hausdorff. La distance de Hausdorff représente la distance maximale entre deux contours ou deux surfaces A et B ; elle est définie par l'équation : 


$$
\operatorname{dh}(\mathrm{A}, \mathrm{B})=\operatorname{Max}\{\mathrm{d}(\mathrm{A}, \mathrm{B}), \mathrm{d}(\mathrm{B}, \mathrm{A})\}
$$

Le calcul de la distance moyenne permet d'apprécier une similitude plus globale entre les contours. Ces distances peuvent être calculées sur des contours, coupe par coupe, ou sur des volumes. La distance radiale est définie comme la distance entre deux points situés sur un même rayon tiré depuis le centre de gravité d'un volume. Elle est plutôt adaptée aux volumes sphériques mais peut être prise en défaut même pour la comparaison des volumes prostatiques lorsque les contours sont asymétriques ou lorsque les vésicules séminales sont incluses dans l'analyse (Figure 2). Dans ce cas le rayon n'est pas perpendiculaire aux deux surfaces et la distance est surestimée [45]. La distance entre les deux points les plus proches est une méthode utilisée notamment par Rao et al. [42] pour l'évaluation des m-reps. Son inconvénient est d'être asymétrique, le résultat dépendant du point de départ (Figure 2). Les auteurs y ont pallié en mesurant les distances les plus proches, en interchangeant les rôles des points de départ et d'arrivée et en poolant les valeurs [42]. Une autre méthode employée par Steenbakkers et al. [48] et Deurloo et al. [19] utilise la distance à partir de la normale de la surface. De la même façon cette méthode n'est pas symétrique (Figure 2); Steenbakkers et al. [48] contournent cette limite en employant la méthode des points les plus proches si la distance à partir de la normale de la surface dépasse une valeur prédéterminée $(2 \mathrm{~cm}$ dans le cas de la délinéation d'un volume cible tumoral macroscopique pulmonaire). Un autre inconvénient des méthodes linéaires est la perte des informations sur la topographie des différences entre les contours (par exemple au niveau de la base et de l'apex prostatiques). Van der Put et al. [50] ont élaboré une méthode de comparaison quantitative à partir de deux champs de vecteurs déterminés à partir des deux contours. La combinaison de ces deux champs de vecteurs permet de relier les points correspondants de chaque contour. Leurs résultats dans la délinéation prostatique montrent que la distance radiale surestime les distances essentiellement au niveau de l'interface avec le rectum, et des vésicules séminales, régions ou la forme de la prostate est la moins sphérique.

Les index volumiques permettent d'apprécier de manière relative et de façon plus globale les différences de délinéation. Les index suivants sont utilisés :

- rapport volumique (rapport des volumes automatique et manuel, dont la valeur optimale est 1)

- "Volume overlap" (rapport du volume de l'intersection sur le volume de l'union, dont la valeur optimale est 1) comme défini dans l'équation [27] : 


$$
V o=\frac{V m \bigcap V a}{V m \bigcup V a}
$$

$\mathrm{VO}=$ volume overlap, $\mathrm{Vm}=$ volume manuel, $\mathrm{Va}=$ volume automatique

- "Volume correctement délinéé" (pourcentage du volume de l'intersection sur le volume manuel dont la valeur optimale est 100) comme défini dans l'équation [6,34] :

$$
V c d=\frac{V m \bigcap V a}{V m} \times 100
$$

$\mathrm{Vcd}=$ volume correctement délinéé $; \mathrm{Vm}=$ volume manuel, $\mathrm{Va}=$ volume automatique

- Index kappa ou coefficient de similarité de Dice (CSD), défini par l'équation :

$$
K i=2 \times \frac{V m \bigcap V a}{V m+V a}
$$

$$
\mathrm{Ki}=\text { Index Kappa } ; \mathrm{Vm}=\text { volume manuel, } \mathrm{Va}=\text { volume automatique }
$$

Les évaluations basées sur les index volumiques (VO,Vcd, Ki) sont plus sensibles aux erreurs de recouvrement que les évaluations métriques. Par exemple, le VO de deux volumes se recouvrant à $85 \%$ ne sera que de 0.74 . De la même manière le VO de 2 cubes de 10 x 10 x 10 voxels décalés de un voxel selon la diagonale du cube sera de $57 \%$ seulement (729/1271) alors que la distance moyenne entre les surfaces est d'environ 1 voxel [27].

Les résultats peuvent également être exprimés en terme de sensibilité et de spécificité, et représentés sous la forme de courbes ROC (Receiver Operating Characteristic). Le «vrai positif»(VP) représente le nombre de voxels communs aux segmentations automatique et manuelle, le «faux positif» $(\mathrm{FP})$ représente les voxels délinéés à tort par rapport à la segmentation manuelle, le «faux négatif» $(\mathrm{FN})$ les voxels non délinéés à tort, et le «vrai négatif » (VN) les voxels non délinéés à juste titre. La sensibilité (Se) et la spécificité ( $\mathrm{Sp})$ peuvent être déterminées par les relations [16,25,47]: 


$$
\begin{aligned}
& S e=\frac{V P}{V P+F N} \\
& S p=\frac{V N}{V N+F P}
\end{aligned}
$$

L'index kappa ou le coefficient de similarité de Dice peut alors s'écrire [16] :

$$
K i=\frac{2 V P}{2 V P+F N+F P}
$$

Le contour manuel est considéré comme le «gold standard» alors que la variabilité inter et intra-observateur dans la délinéation des volumes d'intérêt a été largement étudiée en fonction des localisations tumorales et de l'examen d'imagerie employé [9,10,20,22,43,46,51,52,54]. Pour y pallier Pallotta et al. [35] ont utilisé un fantôme numérique de géométrie connue afin de tester un algorithme de segmentation automatique de la prostate et des volumes d'intérêt pelviens. Ce fantôme numérique simulait des images tomodensitométriques comportant sept régions de niveaux de gris différents. Malgré l'ajout de bruit de Poisson homogène à ces images, le contraste entre les régions restait différent des conditions cliniques, conduisant probablement à une surestimation des capacités de l'algorithme de segmentation. Il n'existe pas de consensus sur les valeurs des index cités plus haut au-delà desquels les contours automatiques peuvent être considérés comme acceptables. L'évaluation des outils de délinéation automatique dans un contexte clinique ou "préclinique », c'est à dire sans utiliser les contours obtenus pour le traitement des patients, devrait donc être réalisée préférentiellement dans le cadre d'études multi-observateurs afin de s'assurer que les contours automatiques sont concordants avec la variabilité inter-observateur.

Ces évaluations multi-observateurs devraient comporter un nombre suffisant de patients et d'observateurs afin d'obtenir des résultats statistiquement fiables. Remeijer et al. [45] ont modélisé l'incertitude de la détermination de la variabilité intra et interobservateur, à partir des données de Rasch et al. [43], comparant les contours prostatiques délinéés par 3 médecins chez 18 patients. L'écart type de ces paramètres est inversement proportionnel à la racine carrée du nombre de patients et au nombre d'observateur. Des différences systématiques peuvent facilement être mises en évidence dans les régions pour lesquelles la variabilité intra 
et inter observateur est la plus faible. A l'inverse les régions comme l'apex prostatique nécessitent un effectif plus important pour déterminer avec précision les variabilités de délinéation. Par exemple la précision de la détermination de la variabilité intra-observateur de l'apex (écart type de $3.5 \mathrm{~mm}$ ) est de $0.7 \mathrm{~mm}$ (19\%) à partir des données de Rasch et al. [43]. Une précision de $5 \%$ nécessiterait de multiplier l'effectif par 9 , soit près de 150 patients au total, ce qui devient plus difficilement réalisable [45].

Les outils de délinéation automatique doivent également être évalués en terme de réduction de temps passé pour la délinéation. L'évaluation de l'influence de ces outils sur la variabilité inter-observateur paraît également être un champ d'investigation important, tout comme l'influence des contours obtenus automatiquement sur les doses délivrées aux volumes cibles et organes à risque.

\section{3) Résultats}

\section{3-1) Cerveau}

Le cerveau a été l'une des premières localisations étudiées, en raison du contraste élevé pour les tissus mous de l'Imagerie par Résonance Magnétique (IRM) employée communément pour la délinéation des volumes d'intérêt. Les méthodes les plus anciennes, basées sur le seuillage, permettent de segmenter des structures simples dans des régions contenant un fort contraste, comme le volume tumoral volume macroscopique (GTV) avec injection de produit de contraste; elles ne seront pas détaillées ici. Mazzara et al. [30] ont évalué deux méthodes de croissance de régions pour la définition du volume tumoral macroscopique (GTV) chez 11 patients traités pour des gliomes de haut grade. Les contours obtenus automatiquement étaient comparés à ceux définis par trois médecins d'après des examens d'IRM avec injection de Gadolinium. Les résultats étaient évalués selon une méthode d'appartenance des pixels aux contours communs réalisés par les médecins. Les volumes communs à la délinéation manuelle étaient pour les deux méthodes de segmentation de seulement $56 \% \pm 6$ et $52 \% \pm 7$, mais ces résultats étaient comparables à la variation interobservateur.

Différentes équipes ont développé des méthodes de délinéation basées sur le recalage élastique avec un atlas. Cuadra et al. [15] ont intégré dans la délinéation cérébrale la déformation induite par la lésion tumorale. Une information a priori de croissance tumorale était intégrée, à partir d'un point de départ défini par l'observateur. Il s'agit cependant d'un 
modèle de croissance radiale, relativement simple et adapté aux volumes réguliers; les résultats étaient satisfaisant chez les 4 patients évalués qui présentaient un méningiome. Des résultats de délinéation automatique du cerveau, du cervelet et du noyau caudé avaient été présenté auparavant par Dawant et al. [16]. Le coefficient de similarité de Dice (CSD) était respectivement de 0.95 et 0.97 pour le cerveau et le cervelet par rapport aux volumes définis par deux observateurs, et de 0.85 pour le noyau caudé. Les résultats inférieurs pour cette dernière structure étaient dus à l'association d'un petit volume et de la faible résolution des images d'IRM, rendant moins pertinent le CSD, la valeur de 0.85 correspondant à une différence de l'ordre du pixel. Bondiau et al. [5] ont modélisé un atlas destiné à la segmentation automatique du tronc cérébral à partir d'images d'IRM artificielles. Cet outil a été comparé au volume défini à partir des contours des 7 experts pour chacun des 6 patients. Les résultats étaient fiables et reproductibles, et s'intégraient dans la variabilité interobservateur. Isambert et al. [25] ont présenté plus récemment les résultats de cet outil de délinéation automatique chez 11 patients; les contours obtenus sur des images IRM pondérées en T1 pour les yeux, les nerfs optiques, le chiasma, l'hypophyse, le cervelet et le tronc cérébral étaient comparés à ceux de deux experts. Les CSD obtenus étaient élevés pour les structures les plus volumineuses (valeur moyenne de 0.81 à 0.85 pour les yeux, le cervelet et le tronc cérébral) mais de façon similaire aux résultats de Dawant et al. [16] les CSD étaient nettement inférieurs pour les structures de plus petit volume (valeur moyenne de 0.3 à 0.41 pour l'hypophyse, les nerfs optiques et le chiasma). Le CSD était supérieur à 0.8 pour des volumes supérieurs à $7 \mathrm{~cm}^{3}$. Ces résultats fiables et reproductibles permettaient l'utilisation de cet outil de délinéation automatique pour les globes oculaires, le cervelet et le tronc cérébral [25].

\section{3-2) Voies aéro-digestives supérieures.}

L'élaboration d'un atlas à partir de données scanographiques de 45 patients est détaillée dans Commowick et al [14]. Les volumes d'intérêt étaient les aires ganglionnaires cervicales II, III et IV selon la classification internationale, les glandes parotides et sous maxillaires. L'évaluation a été menée chez 12 des 45 patients ayant servi à l'élaboration de l'atlas (les données de chaque patient étudié sont alors exclues de l'atlas). La sensibilité et la spécificité étaient respectivement de 0.82 et 0.86 . Des résultats plus détaillés de cet outil ont été publiés par Sims et al. [47] dans le cadre du projet "Methods and Advanced Equipment for 
Simulation and Treatment in Radio Oncology" (MAESTRO). Cette évaluation a concerné la délinéation du tronc cérébral, des parotides et de la mandibule chez 13 patients. Les contours obtenus étaient comparés à ceux d'experts appartenant à deux sites. Les experts devaient définir les volumes d'intérêt manuellement (site 1) ou corriger si besoin les contours obtenus automatiquement (site 2). La délinéation automatique aboutissait à une « sous-estimation »du tronc cérébral en raison d'une extension craniale insuffisante des images scanographiques ayant servi à l'élaboration de l'atlas, et une «sur-estimation» des parotides qui sera corrigée par une opération morphomathématique d'érosion. La concordance avec les contours des experts était satisfaisante avec un CSD moyen de toutes les structures de $0.68 \pm 0.25$ et 0.82 \pm 0.13 selon les sites 1 et 2 respectivement. Les CSD s'étalaient de $0.58 \pm 0.2$ (tronc cérébral) à $0.78 \pm 0.06$ (mandibule) pour le site 1 , et de $0.77 \pm 0.07$ (tronc cérébral) à $0.86 \pm 0.07$ (parotide) pour le site 2 [47]. Ces erreurs systématiques dans la délinéation des glandes parotides semblent avoir été corrigées dans la dernière version évaluable (version 4.2), comme l'a souligné un travail présenté en communication orale durant le congrès de la Société Française de Radiothérapie Oncologique en 2009 [41]. Ce travail portait sur 11 cas de cancers des voies aéro-digestives supérieures dont les aires II, III, IV et V et les parotides étaient délinées. Il existait une bonne corrélation entre les contours des 3 oncologues radiothérapeutes mais une discordance avec les volumes proposés par le logiciel. La différence portait essentiellement sur les niveaux de transition entre les aires ganglionnaires (différence supprimée en cas d'unification des aires II, III et IV) et sur les aires V. Les parotides avaient une délinéation d'emblée acceptable. Par ailleurs, les auteurs notaient l'absence de gain de temps compte tenu de la somme des corrections nécessaires.

Zhang et al. [56] ont présenté leurs résultats dans le cadre de la radiothérapie guidée par l'image. Un atlas était développé à partir des données du scanner de planification dosimétrique. Une évaluation a été réalisée chez 7 patients et 32 images. Les résultats obtenus pour le tronc cérébral, la mœlle épinière, la mandibule, les parotides et les aires ganglionnaires cervicales étaient reproductibles, avec un CSD variant pour l'ensemble de ces structures de 0.65 à 0.9. Wang et al. [53] ont mené un travail similaire, en évaluant le recalage élastique entre les examens de tomodensitométrie de planification et réalisés au cours du traitement (scanner sur rail). Les contours obtenus étaient comparés à ceux définis manuellement ou simplement corrigés après délinéation automatique. Les résultats chez 8 patients (100 images au total) montraient un VO moyen de 73 à $86 \%$ pour les parotides par rapport au contour manuel et de 98 à 100\% par rapport aux contours corrigés. Le VO moyen variait de 94 à 97\% pour le volume cible anatomo-clinique par rapport aux volumes corrigés. 
Les résultats respectifs de plusieurs algorithmes de recalage élastique sont détaillés dans Castadot et al. [8].

\section{3-3) Pelvis}

De nombreuses méthodes de segmentation automatique ont été évaluées dans le cadre de la radiothérapie pelvienne. Une méthode basée sur l'algorithme de la ligne de partage des eaux a été présentée pour le rectum, la vessie et les vésicules séminales d'après des images scanogaphiques avec des résultats satisfaisants au vu de sa simplicité (1.2 à $1.7 \mathrm{~mm}$ de distance moyenne entre les contours automatiques et manuels pour le rectum et la vessie) [6]. Les résultats d'une segmentation par extension de région de la prostate, du rectum et de la vessie sur des images tomodensitométriques ont été publiés par Mazonakis et al. [29] mais il ne s'agit pas à proprement parler d'une méthode automatique, l'utilisateur devant choisir manuellement les seuils de niveaux de gris.

Plus récemment plusieurs équipes ont présenté des résultats basés sur des modèles déformables et des atlas pour la délinéation automatique de la prostate et/ou des organes à risque. Des modèles déformables ont été élaborés pour la délinéation prostatique sur des images échographiques [2,3,21]. Pekar et al. [39] ont présenté les résultats d'une segmentation automatique basée sur des modèles déformables de la vessie, du rectum et des têtes fémorales d'après des images tomodensitométriques. Les contours obtenus étaient comparés à ceux réalisés par un expert chez 40 patients pour le rectum et la vessie, et 15 patients pour une tête fémorale. Après correction manuelle des écarts les plus importants, les distances moyennes et maximales entre les contours automatiques et manuels de la vessie étaient respectivement de $1.47 \pm 0.45 \mathrm{~mm}$ et $8 \pm 1.5 \mathrm{~mm}$. Ces valeurs étaient respectivement de $1.6 \pm 0.4 \mathrm{~mm}$ et $7.6 \pm 1.8$ pour le rectum, et de $0.93 \pm 0.1 \mathrm{~mm}$ et $5 \pm 1 \mathrm{~mm}$ pour une tête fémorale. D'autres équipes ont utilisé un modèle déformable cette fois pour la délinéation de la prostate, notamment d'après des images IRM. Les rapports de volume, VO et Vcd étaient respectivement de $1.13 \pm 0.1,0.78 \pm 0.05$ et $94.75 \pm 3.3$ par rapport à la délinéation manuelle chez 24 patients dans [37]. Un VO moyen de $0.78 \pm 0.05$ correspond à un CSD de $0.88 \pm 0.04$ [28,37]. Le contraste élevé des images IRM a permis d'utiliser des méthodes d'extension de région pour le rectum et la vessie. Pour le rectum, les rapports de volume, VO et Vcd étaient respectivement de $0.97 \pm 0.1,0.78 \pm 0.06$ et $86.52 \pm 5$, et de $0.95 \pm 0.03,0.88 \pm 0.03$ et $91.3 \pm 3$ pour la vessie [37]. Has et al. ont détaillé l'élaboration de leur méthode dans [23]; les résultats spécifiques de la segmentation de la prostate, du rectum, de la vessie et des têtes fémorales 
d'après des images scanographiques sont présentés dans [24]. Une évaluation qualitative était réalisée par 6 experts chez 39 patients. Les contours automatiques devaient être classés comme excellent, bon, acceptable ou non acceptable. Les contours prostatiques ont été considérés comme bon, acceptable et non acceptable dans 45\%, 30\% et $15 \%$ des cas respectivement. Les résultats sont similaires pour la vessie, avec $36 \%$ et $42 \%$ de contours considérés comme excellent ou bon, mais sont inférieurs pour le rectum et les têtes fémorales. L'évaluation quantitative menée par 7 experts chez 7 patients montre des résultats qui s'inscrivent dans la variabilité inter observateur pour la prostate et la vessie, et là encore inférieurs pour le rectum. Une méthode basée sur un atlas prostatique incluant les vésicules séminales est proposée par Klein et al. [28]. L'évaluation est réalisée sur des images IRM chez 50 patients. Le CSD médian est de 0.85 , proche du CSD médian interobservateur de 0.87. Les auteurs soulignent l'importance de la constitution de l'atlas qui doit se rapprocher des conditions cliniques et comporter des variations anatomiques.

\section{3-4) Autres critères d'évaluation}

L'un des principaux buts des outils de segmentation automatique est de réduire le temps de délinéation. Dans Isambert et al. [25] le temps moyen de segmentation automatique des structures cérébrales était de 7 à 8 minutes sans intervention humaine, contre environ trente minutes à une heure pour la délinéation manuelle de ces mêmes structures. Concernant les localisations pelviennes un gain de temps peut également être obtenu [24,31,39]. La segmentation des organes à risque est obtenue en 10 à 15 minutes interactions comprises [39], et en une minute pour la prostate et les organes à risque contre 25 minutes manuellement [24]. Une segmentation rénale est obtenue en 7 minutes dans [40,42]. Dans un contexte de radiothérapie guidée par l'image Wang et al. [53] rapportent des durées de 2 et 3 minutes pour les localisations des voies aéro-digestives supérieures et thoraciques. Il est parfois difficile dans la littérature de déterminer si le temps de correction et d'interaction est comptabilisé dans la durée de segmentation automatique.

Peu de données sont disponibles concernant l'influence de la segmentation automatique sur la variabilité inter observateur. Chao et al. [12] ont montré que cette variabilité pouvait être significativement diminuée entre 8 médecins dans la délinéation des CTV et organes à risque chez deux patients présentant une tumeur des voies aéro-digestives supérieures avec envahissement ganglionnaire. Les VO moyens des différents CTV s'étalaient de 0.53 à 0.65 et de 0.91 à 0.93 respectivement sans et avec segmentation automatique dans le cas de la tumeur 
de base de langue $(\mathrm{p}<0.001)$. Ces valeurs étaient de 0.32 à 0.61 et 0.89 à 0.92 dans le cas de la tumeur du cavum $(\mathrm{p}<0.001)$. Dans les deux cas la concordance de la délinéation des organes à risque était également améliorée. La segmentation automatique peut également diminuer la variabilité de la délinéation de la glande mammaire. Dans Reed et al. [44] les volumes définis par 8 médecins chez 10 patientes d'après des images scanographiques étaient comparés, avec et sans aide à la segmentation. La délinéation de chaque médecin était confrontée à un volume « commun » à 5 des 8 médecins. La distance moyenne était réduite de 1.6 à $1 \mathrm{~mm}(\mathrm{p}=0.049)$; le CSD était augmenté de 0.92 à $0.94(\mathrm{p}=0.049)$ et la durée de délinéation diminuée de $30 \%$ $(\mathrm{p}=0.028)$. Une diminution de la variabilité interobservateur ne préjuge cependant pas d'une amélioration de la précision, des erreurs systématiques des outils de segmentation pourraient conduire à s'écarter des recommandations de délinéation. La finalité des aides automatiques est leur utilisation clinique et quelques équipes ont publié des résultats préliminaires sur leur évaluation dosimétrique. Les écarts de délinéation sont lissés par l'ajout de marges lors de la création du volume cible planifié; ces résultats dosimétriques sont dépendants des techniques de radiothérapie $[4,38]$.

\section{4) $\underline{\text { Conclusion }}$}

Les développements informatiques ont permis de réaliser récemment des progrès considérables dans l'évolution des outils de segmentation automatique; ces progrès ont été rendus nécessaires par l'essor de la radiothérapie conformationnelle et guidée par l'image. Les résultats cliniques sont pour la plupart préliminaires. Dans la littérature le degré d'interaction nécessaire est parfois peu détaillé ou important. Alors que la présence d'outils de délinéation automatique fait partie des arguments commerciaux mis en avant par les industriels lors du choix d'une station de dosimétrie ou d'un système de radiothérapie guidée par l'image, les résultats de ces outils sont parfois insuffisamment évalués. Ils nécessitent une évaluation rigoureuse pré-clinique, multicentrique, portant sur leur fiabilité, leur concordance avec les recommandations de délinéation, le gain de temps apporté et leur reproductibilité. Leur apport dans la réduction des écarts inter-observateurs paraît être un champ d'investigation important. Leurs développements actuels laissent penser que ces outils feront prochainement partie intégrante de notre environnement et deviendront des aides précieuses à la délinéation, sans toutefois pouvoir remplacer l'expertise humaine ni la confrontation multidisciplinaire. 
1) Besl P, McKay N. A method for registration of 3D shapes, IEEE Trans. Pattern Analysis and Machine Intelligence. 1992; 14(2): 239-255

2) Betrouni N, Palos G, Coulanges M, Vermandel M, Maouche S, Rousseau J. A method to register intra-treatment ultrasound images to pre-treatment images of prostate. Conf Proc IEEE Eng Med Biol Soc. 2004; 3: 1741-4

3) Betrouni N, Pasquier D, Dewalle AS, Jounwaz R, Dubois P, Lopes R et al. Ultrasound image registration for patient setup in conformal radiotherapy of prostate cancer. Conf Proc IEEE Eng Med Biol Soc. 2006; 1: 3795-8.

4) Beyer GP, Velthuizen RP, Murtagh FR, Pearlman JL. Technical aspects and evaluation methodology for the application of two automated brain MRI tumor segmentation methods in radiation therapy planning. Magn Reson Imaging. 2006; 24: 1167-78

5) Bondiau PY, Malandain G, Chanalet S, Marcy PY, Habrand JL, Fauchon F et al. Atlasbased automatic segmentation of MR images: validation study on the brainstem in radiotherapy context. Int J Radiat Oncol Biol Phys. 2005; 61: 289-98

6) Bueno G, Fisher M, Burnham K. Automatic segmentation of clinical structures for RTP: Evaluation of a morphological approach. In: Proceedings of Medical Image Understanding and Analysis (MIUA ’01). Birmingham, UK: BMVA Press; 2001; p. 73-76.

7) Canny J. A Computational Approach To Edge Detection. IEEE Trans. Pattern Analysis and Machine Intelligence. 1986: 8: 679-714

8) Castadot P, Lee JA, Parraga A, Geets X, Macq B, Grégoire V. Comparison of 12 deformable registration strategies in adaptive radiation therapy for the treatment of head and neck tumors. Radiother Oncol. 2008; 89: 1-12

9) Cattaneo GM, Reni M, Rizzo G, Castellone P, Ceresoli GL, Cozzarini C et al. Target delineation in post-operative radiotherapy of brain gliomas: interobserver variability and impact of image registration of MR(pre-operative) images on treatment planning CT scans. Radiother Oncol. 2005; 75: 217-23. 
10) Cazzaniga LF, Marinoni MA, Bossi A, Bianchi E, Cagna E, Cosentino D et al. Interphysician variability in defining the planning target volume in the irradiation of prostate and seminal vesicles. Radiother Oncol 1998;47: 293-6.

11) Chaney EL, Pizer SM. Autosegmentation of images in radiation oncology. J Am Coll Radiol. 2009; 6: 455-8

12) Chao KS, Bhide S, Chen H, Asper J, Bush S, Franklin G et al. Reduce in variation and improve efficiency of target volume delineation by a computer-assisted system using a deformable image registration approach. Int J Radiat Oncol Biol Phys. 2007; 68: 1512-21

13) Cohen LD, Cohen I. (1993). Finite element methods for active contour models and balloons for 2D and 3D images. IEEE Trans. Pattern Anal. Machine Intelligence. 1993; 15: $1131-47$

14) Commowick O, Grégoire V, Malandain G. Atlas-based delineation of lymph node levels in head and neck computed tomography images. Radiother Oncol. 2008; 87: 281-9

15) Cuadra MB, Pollo C, Bardera A, Cuisenaire O, Villemure JG, Thiran JP. Atlas-based segmentation of pathological MR brain images using a model of lesion growth. IEEE Trans Med Imaging 2004; 23: 1301-14

16) Dawant BM, Hartmann SL, Thirion JP, Maes F, Vandermeulen D, Demaerel P. Automatic 3D segmentation of internal structures of the head in MR images using a combination of similarity and free-form transformations: Part I, methodology and validation on normal subjects. IEEE Trans Med Imaging 1999; 18: 909 -916

17) Deriche R. Using Canny's criteria to derive a recursively implemented optimal edge detector. Int Journal of Computer Vision. 1987; 1(2): 167-87

18) Derraz F, Peyrodie L, Pinti A, Taleb-Ahmed A., Chick L. Multiple sclerosis segmentation based geometric active contour model, Edition Tech Science Press, ICCES. 2009; 557 
19) Deurloo KE, Steenbakkers RJ, Zijp LJ, de Bois JA, Nowak PJ, Rasch CR et al. Quantification of shape variation of prostate and seminal vesicles during external beam radiotherapy. Int J Radiat Oncol Biol Phys. 2005; 61: 228-38.

20) Fiorino C, Reni M, Bolognesi A, Cattaneo GM, Calandrino R. Intra- and inter-observer variability in contouring prostate and seminal vesicles: implications for conformal treatment planning. Radiother Oncol 1998; 47: 285-92.

21) Ghanei A, Soltanian-Zadeh H, Ratkewicz A, Yin F. A three-dimensional deformable model for segmentation of human prostate from ultrasound images. Med Phys. 2001; 28: 2147-53

22) Giraud P, Elles S, Helfre S, De Rycke Y, Servois V, Carette MF et al. Conformal radiotherapy for lung cancer: different delineation of the gross tumor volume (GTV) by radiologists and radiation oncologists. Radiother Oncol 2002; 62: 27-36.

23) Haas B, Coradi T, Scholz M, Kunz P, Huber M, Oppitz U et al. Automatic segmentation of thoracic and pelvic CT images for radiotherapy planning using implicit anatomic knowledge and organ-specific segmentation strategies. Phys Med Biol. 2008; 53: 1751-71

24) Huyskens DP, Maingon P, Vanuytsel L, Remouchamps V, Roques T, Dubray B et al. A qualitative and a quantitative analysis of an auto-segmentation module for prostate cancer. Radiother Oncol. 2009; 90: 337-45.

25) Isambert A, Dhermain F, Bidault F, Commowick O, Bondiau PY, Malandain G, Lefkopoulos D. Evaluation of an atlas-based automatic segmentation software for the delineation of brain organs at risk in a radiation therapy clinical context. Radiother Oncol 2008; 87: 93-99

26) Kass M, Witkin A, Terzopoulos D. Snakes: Active contour models. Int. J. Comput. Vision. 1988; 1: 321-331

27) Kelemen A, Szekely G, Gerig G. Elastic model-based segmentation of 3-D neuroradiological data sets. IEEE Trans Med Imaging 1999; 18: 828-839 
28) Klein S, van der Heide UA, Lips IM, van Vulpen M, Staring M, Pluim JP. Automatic segmentation of the prostate in 3D MR images by atlas matching using localized mutual information. Med Phys. 2008; 35: 1407-17

29) Mazonakis M, Damilakis J, Varveris H. Image segmentation in treatment planning for prostate cancer using the region growing technique. Br J Radiol 2001; 74: 243-248

30) Mazzara GP, Velthuizen RP, Pearlman JL, Greenberg HM, Wagner H. Brain tumor target volume determination for radiation treatment planning through automated MRI segmentation. Int J Radiat Oncol Biol Phys 2004; 59: 300-12.

31) McBain CA, Moore CJ, Green MM, Price G, Sykes JS, Amer A, et al. Early clinical evaluation of a novel three-dimensional structure delineation software tool (SCULPTER) for radiotherapy treatment planning. Br J Radiol. 2008; 81: 643-52

32) McInerney T, Terzopoulos D. Deformable models in medical image analysis: a survey. Med Image Anal. 1996; 1: 91-108

33) Merck D, Tracton G, Saboo R, Levy J, Chaney E, Pizer S et al. Training models of anatomic shape variability. Med Phys. 2008; 35: 3584-96

34) Metz C. Basic principles of ROC analysis. Semin Nucl Med 1978; 8: 283-298

35) Pallotta S, Bucciolini M, Russo S, Talamonti C, Biti G. Accuracy evaluation of image registration and segmentation tools used in conformal treatment planning of prostate cancer. Comput Med Imaging Graph. 2006; 30: 1-7

36) Palos Ladeiro G. Thèse de Doctorat en Automatique et informatique industrielle. Recalage non rigide d'images médicales: application aux images abdominales multimodalités. Université Lille I. 2004 
37) Pasquier D, Lacornerie T, Vermandel M, Rousseau J, Lartigau E, Betrouni N. Automatic segmentation of pelvic structures from magnetic resonance images for prostate cancer radiotherapy.Int J Radiat Oncol Biol Phys. 2007; 68: 592-600

38) Pasquier D, Lacornerie T, Betrouni N, Vermandel M, Rousseau J, Lartigau E. Évaluation dosimétrique d'un outil de délinéation automatique des organes pelviens à partir d'images IRM pour la radiothérapie du cancer prostatique. Cancer Radiother. 2008; 12: 323-30

39) Pekar V, McNutt TR, Kaus MR. Automated model-based organ delineation for radiotherapy planning in prostatic region. Int J Radiat Oncol Biol Phys. 2004; 60: 973-80

40) Pizer SM, Fletcher PT, Joshi S, Gash AG, Stough J, Thall A et al. A method and software for segmentation of anatomic object ensembles by deformable m-reps. Med Phys. 2005; 32: $1335-45$

41) Pointreau Y, Bera G, Barillot I. Aide à la délinéation : Quels outils pratiques ? Cancer Radiother 2009; 13: 600-5.

42) Rao M, Stough J, Chi YY, Muller K, Tracton G, Pizer SM, et al. Comparison of human and automatic segmentations of kidneys from CT images. Int J Radiat Oncol Biol Phys. 2005; 61: $954-60$

43) Rasch C, Barillot I, Remeijer P, Touw A, van Herk M, Lebesque JV. Definition of the prostate in CT and MRI: a multi-observer study. Int J Radiat Oncol Biol Phys 1999; 43: 5766.

44) Reed VK, Woodward WA, Zhang L, Strom EA, Perkins GH, Tereffe W et al. Automatic segmentation of whole breast using atlas approach and deformable image registration. Int $\mathbf{J}$ Radiat Oncol Biol Phys. 2009; 73: 1493-500

45) Remeijer P, Rasch C, Lebesque J, Van Herk M. A general methodology for threedimensional analysis of variation in target volume delineation. Med Phys. 1999: 26: 931-40 
46) Seddon B, Bidmead M, Wilson J, Khoo V, Dearnaley D. Target volume definition in conformal radiotherapy for prostate cancer: quality assurance in the MRC RT-01 trial. Radiother Oncol 2000; 56: 73-83.

47) Sims R, Isambert A, Grégoire V, Bidault F, Fresco L, Sage J et al. A pre-clinical assessment of an atlas-based automatic segmentation tool for the head and neck. Radiother Oncol. 2009; 93: 474-8.

48) Steenbakkers RJ, Duppen JC, Fitton I, Deurloo KE, Zijp LJ, Comans EF et al. Reduction of observer variation using matched CT-PET for lung cancer delineation: a three-dimensional analysis. Int J Radiat Oncol Biol Phys. 2006; 64: 435-48.

49) Terzopoulos D, Fleischer K. Deformable models. The Visual Computer. 1988; 4: 306331

50) Van der Put RW, Raaymakers BW, Kerkhof EM, van Vulpen M, Lagendijk JJ. A novel method for comparing 3D target volume delineations in radiotherapy. Phys Med Biol. 2008; 53: 2149-59.

51) Van de Steene J, Linthout N, de Mey J, Vinh-Hung V, Claassens C, Noppen M et al. Definition of gross tumor volume in lung cancer: inter-observer variability. Radiother Oncol 2002; 62: 37-49

52) Vorwerk H, Beckmann G, Bremer M, Degen M, Dietl B, Fietkau R, et al. The delineation of target volumes for radiotherapy of lung cancer patients Radiother Oncol. 2009; 91: 455-60

53) Wang H, Garden AS, Zhang L, Wei X, Ahamad A, Kuban DA et al. Performance evaluation of automatic anatomy segmentation algorithm on repeat or four-dimensional computed tomography images using deformable image registration method. Int $\mathbf{J}$ Radiat Oncol Biol Phys. 2008; 72: 210-9.

54) Weltens C, Menten J, Feron M, Bellon E, Demaerel P, Maes F, et al. Interobserver variations in gross tumor volume delineation of brain tumors on computed tomography and impact of magnetic resonance imaging. Radiother Oncol. 2001; 60: 49-59. 


\section{5) www.siriade.org}

56) Zhang T, Chi Y, Meldolesi E, Yan D. Automatic delineation of on-line head-and-neck computed tomography images: toward on-line adaptive radiotherapy. Int J Radiat Oncol Biol Phys. 2007; 68: 522-30. 

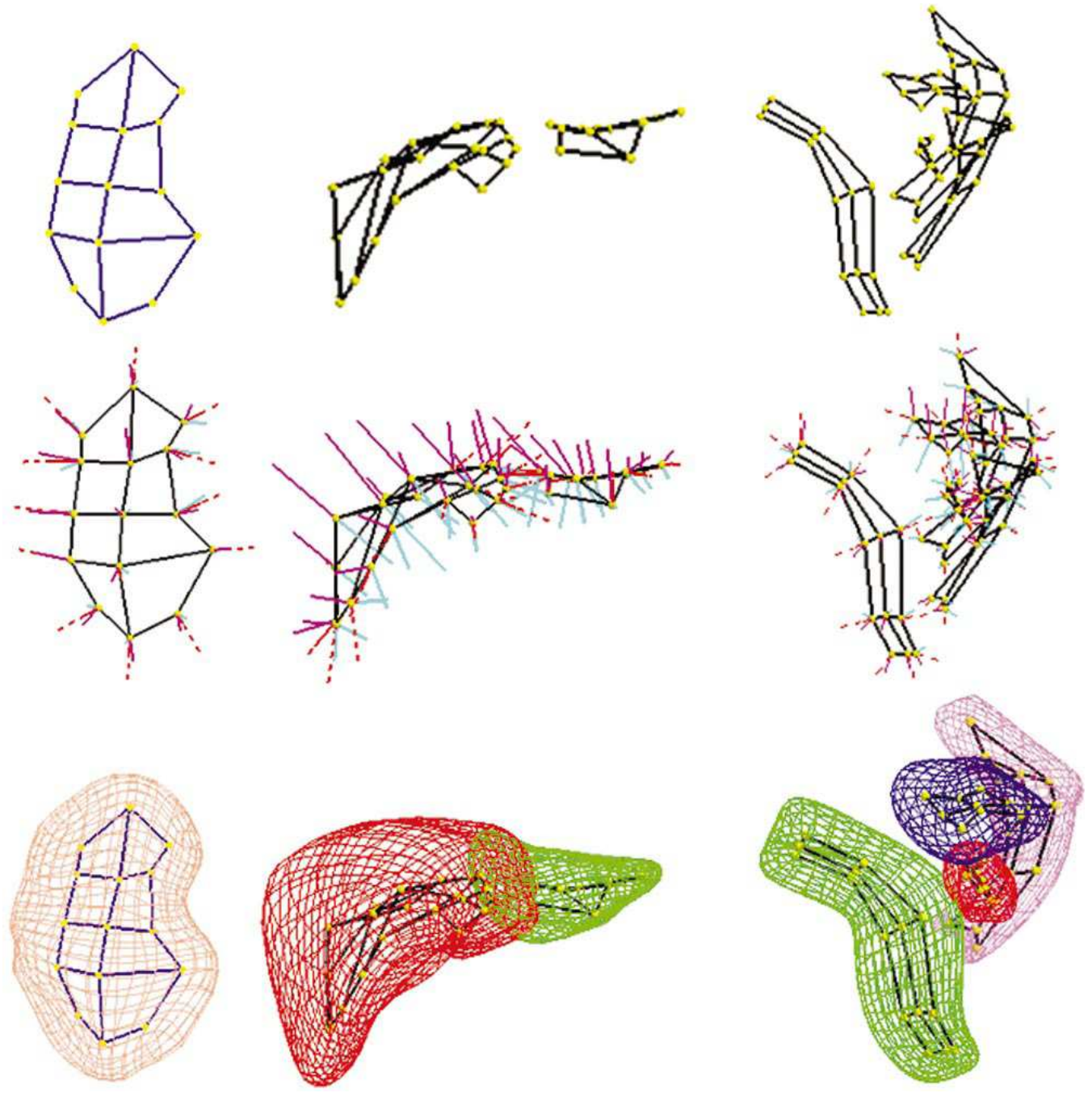

Figure 1. m-reps du rein, du foie et du pelvis masculin. Ligne supérieure : réseau d'atomes centraux. Ligne centrale: réseau d'atomes avec leurs rayons. Ligne inférieure : surface des modèles associées aux atomes, d'après [40]

Figure 1. M-reps for kidney, liver, and male pelvis. Top row: mesh of atom hubs; middle row; mesh atoms including spokes; bottom row: the implied boundaries shown with atom meshes from $[40]$ 


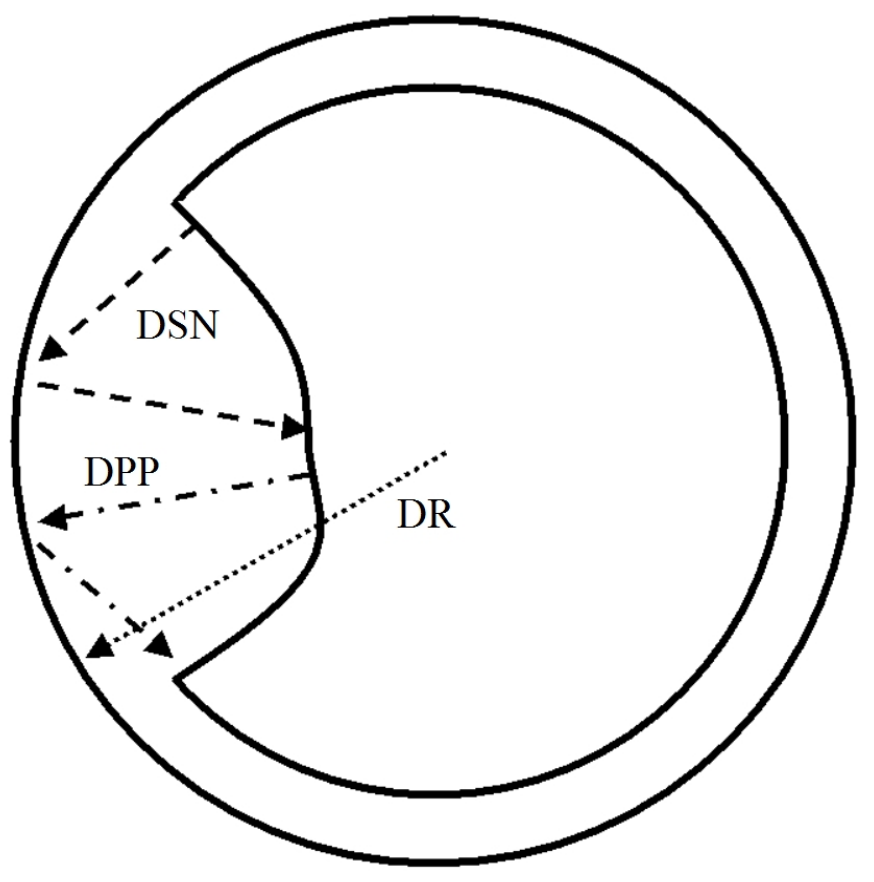

Figure 2. Inconvénients des méthodes de comparaison linéaires dans le cas de contours non symétriques. La distance radiale (DR) peut sur ou sous estimer la distance si la droite n'est pas perpendiculaire à l'un ou l'autre des contours. Les méthodes des distances des points les plus proches (DPP) et de la surface normale (DSN) ne sont pas symétriques et donnent des résultats différents en fonction du point de départ.

Figure 2. Disadvantages of line-based comparison methods when using asymmetric contours. The radial distance (RD) may over- or underestimate the true distance if the straight line is not perpendicular to one or other of the contours. The closest-point and surface normal distance methods are not symmetric and their outcomes vary as a function of the starting point. 